\title{
Acinetobacter endocarditis: a rare nosocomial infection of native heart valves
}

\author{
Shervin Shokouhi ${ }^{1}$, Ghodsieh Kamrani ${ }^{2}$, Iman Ghasemzadeh ${ }^{1,3}$, Mana Baziboroun ${ }^{1,4 *}$ \\ ${ }^{I}$ Infectious Diseases and Tropical Medicine Research Center, Shahid Beheshti University of Medial Sciences, \\ Tehran, Iran \\ ${ }^{2}$ Cancer Research Center, Health Research Institute, Babol University of Medical Sciences, Babol, Iran \\ ${ }^{3}$ HIV/STI Surveillance Research Center, WHO Collaborating Center for HIV Surveillance, Institute for Futures \\ Studies in Health, Kerman University of Medical Sciences, Kerman, Iran \\ ${ }^{4}$ Infectious Diseases and Tropical Medicine Research Center, Health Research Institute, Babol University of \\ Medical Sciences, Babol, Iran
}

Received: January 2021, Accepted: August 2021

\begin{abstract}
Acinetobacter baumannii is an opportunistic bacterial pathogen predominantly associated with hospital-acquired infections. Here we present a case of infective endocarditis of native Mitral and Aorta valves caused by A. baumannii in a 73-year-old man. He underwent surgical excision and Pathologic specimen showed A. baumannii growth after 48 hours that was extensively drug-resistant (XDR). He was treated with colistin and tigecycline. Finally, he discharged with no important complication. To our best knowledge, it is the first case of Acinetobacter endocarditis has ever been reported in Iran. Although XDR A. baumannii is a life-threatening pathogen, proper and timely treatment can be life-saving.
\end{abstract}

Keywords: Acinetobacter baumannii; Endocarditis; Heart valve; Nosocomial infection; Multidrug resistance

\section{INTRODUCTION}

Acinetobacter baumannii is an opportunistic bacterial pathogen predominantly associated with hospital-acquired infections and potential for substantial antimicrobial resistance (1). It is widely distributed in the environment and in the hospital setting such as on environmental surfaces, the health care workers hands, mechanical ventilators and dialysis machines. Most common infections include pneumonia, bacteremia, meningitis, skin, soft tissue and urinary tract infection (2).
Reports of Acinetobacter endocarditis are very rare and most cases have been noted to involve prosthetic valve. It occurs mainly in hospitalized patients with predisposing factors (3). Native valve endocarditis caused by Acinetobacter is acute and aggressive and is more likely to be fatal than the prosthetic valve involvement (4). Increasing resistance to antibiotics, makes this infection difficult to treat and associated with higher mortality (3).

To our best knowledge, it is the first case of Acinetobacter endocarditis has ever been reported in Iran.

*Corresponding author: Mana Baziboroun, MD, Infectious Diseases and Tropical Medicine Research Center, Shahid Beheshti University of Medial Sciences, Tehran, Iran; Infectious Diseases and Tropical Medicine Research Center, Health Research Institute, Babol University of Medical Sciences, Babol, Iran. Telefax: +98-11-32238284Ｅmail: manabaziboron@gmail.com 


\section{CASE PRESENTATION}

A 73-year-old man admitted to Erfan hospital, Tehran, Iran, with fever, generalized weakness and dyspnea. He had history of cholecystectomy duoe to cholecystitis 56 days ago. Following surgery, he gradually developed shortness of breath but he did not seek care. Continued dyspnea and addition of fever, took him to the emergency room. He had history of hypertension and no history of addiction or diabetes mellitus. At presentation, temperature was $38^{\circ} \mathrm{C}$, respiratory rate 18 breaths per minute, heart rate 84 beat per minute, blood pressure 130/70 $\mathrm{mmHg}$ and oxygen saturation $94 \%$. Heart auscultation indicated early systolic and diastolic murmur. Laboratory test showed a total leukocyte count of 8700 with $90 \%$ polymorphs and anemia ( $\mathrm{Hb} 7 / 5 \mathrm{gr} / \mathrm{dl})$. His erythrocyte sedimentation rate was $19 \mathrm{~mm} / \mathrm{h}$ and his serum level of C-reactive protein was $29 \mathrm{mg} / \mathrm{dl}$. Blood urea, serum creatinin, sodium and potassium were in normal ranges. His chest CT scan demonstrated mild plural effusion in both lungs. In transthoracic echocardiography he had moderate MR and moderate TR with a mobile calcified mass on aorta valve. Transesophageal echocardiography indicated Mitral valve thickening with severe regurgitation and perforation of anterior leaflet; Aortic valve thickening with severe insufficiency and perforation of non-coronary cusp (NCC); mobile mass measuring about $6 \times 7 \mathrm{~mm}$ on NCC and mild pericardial effusion posterior to right atrium (Fig. 1). Three sample of blood culture by hourly intervals was negative. After 2 weeks of treatment with vancomycin, ampicillin/sulbactam and gentamicin, there was no improvement in the patient. So, he underwent surgical excision and valves replacement that revealed vegetation on aorta and mitral valves. Pathologic specimen showed severe neutrophilic infiltration with areas of necrosis, granulation tissue, fibrous deposition and bacterial colonization (Fig. 2). Tissue sample was also placed in sterile screw-cap container and drops of sterile saline were added to keep it moist. It was transported to the microbiological laboratory in 15 minutes. The sample was cultured on MacConkey agar and chocolate blood agar and after 48 hours Gram-stained smear showed Gram-negative pleomorphic coccobacilli which grew as non lactose fermenting colonies. The organism was oxidase negative, catalase positive and non mobile. The isolate was identified as Acinetobacter baumannii complex based on glucose oxidation and Triple Suger Iron (TSI) test.

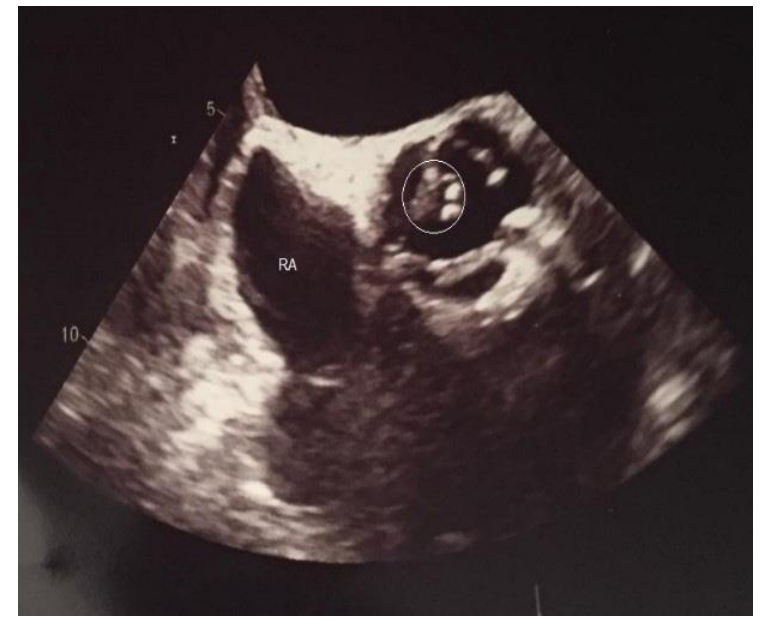

Fig. 1. Transesophageal echocardiography shows $6 \times 7 \mathrm{~mm}$ vegetation (white circle) on the NCC

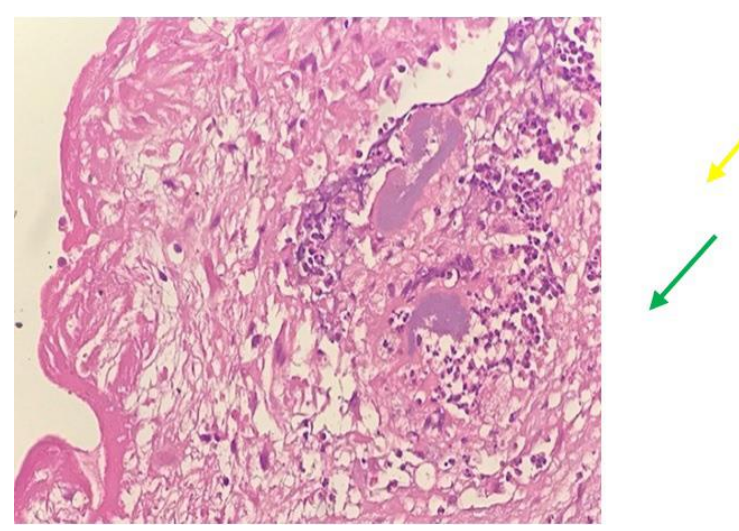

Fig. 2. Histopathological section of endocardial tissue demonstrating neutrophilic infiltration (yellow arrow)and Acinetobacter colonization (green arrow) in hematoxylinand eosin staining (magnification 400)

Antimicrobial susceptibility testing was performed using the Kirby-Bauer disc diffusion method as per clinical and laboratory standard institute recommendation and based on that, the organism was XDR and was only sensitive to colistin. According to the antibiotic susceptibility pattern, medical treatment of the patient changed to intra-venous colistin and tigecycline. Finally, he discharged 42 days after operation and antibiotic therapy with no important complication and no vegetation on fallow up echocardiography.

\section{DISCUSSION}

Acinetobacter baumannii has a high incidence among immunocompromised patients, in particular, 
patients with a history of prolonged ( $>90$ days) hospital stay (5). Other risk factors for developing Acinetobacter infections include immune suppression, older age, presence of co-morbidity, major trauma, invasive procedures, previous antibiotic use and presence of indwelling catheters or mechanical ventilation (6). In the case of endocarditis with rare organisms, several case reports have been published, like a case of IE due to Facklamia hominis in an adult patient with rheumatic mitral stenosis (7) or a rare case of Corynebacterium striatum endocarditis on a bioprosthetic aortic valve (8). There are few reports of endocarditis duo to A. baumannii. In most of these reports, underlying heart disease as a risk factor has been reported and is often seen in patients with prosthetic valve or heart valve disorder, as reported by Qian Chen in 2015, Mauro Sturiale in 2016 and S Senthil Kumar in 2008 (9-11). Our patient had no history of heart disorder but he had a history of cholecystectomy and hospitalization 56 days before.

Despite advances in the treatment of infective endocarditis, it remains challenging (3). In recent years, MDR pathogens have increasingly become a serious global concern with regard to both community-acquired and nosocomial infections. A. baumannii has been designated as a "red alert" pathogen, duoe to its extensive antibiotic resistance spectrum; making selection of an appropriate empirical treatment difficult, so antibiotic therapy should be performed following antimicrobial susceptibility testing. Nevertheless, delay in beginning correct treatment may have adverse effects on patient health (6). In most cases of reported Acinetobacter endocarditis, the pathogen was $\operatorname{MDR}(9,10)$ and treatment was performed based on antibiotic susceptibility testing, but in the case of Acinetobacter endocarditis reported by S. Senthil Kumer in 2008 in India, the isolate was found to be sensitive to a majority of the antibiotics tested (11). Differences in antibiotic susceptibility patterns may be explained by differences in time and place where the studies were performed.

Yoshinori Sato Showed tigecycline killed MDR Acinetobacter more effectively than colistin, although colistin and tigecycline are both considered effective against MDR Acinetobacter (12). These results are consistent with the results reported by Khoshrood S in southwestern Iran who reported $87 \%$ and $12.8 \%$ of Acinetobacter isolates were MDR and XDR, respectively. Colistin and tigecycline with $2.8 \%$ and $45.7 \%$ resistance rates were the most effective antibiotics
(13). Fereshteh Ezadi reported $38 \%$ and $61.9 \%$ of Acinetobacter isolates were carbapenem-susceptible and carbapenem-resistant, respectively. In addition, $94.36 \%$ of isolates were susceptible to colistin (14). Our patient had negative blood culture results, so initially he was treated with vancomycin, ampicillin/ sulbactam and gentamicin but after the culture of tissue specimen was positive for XDR A. baumannii, according to the result of antimicrobial susceptibility testing, treatment was changed to a combination of colistin and tigecycline.

Duoe to the poor prognosis of critically ill patients who acquire Acinetobacter infections, attribution to a definite mortality rate is difficult; however the mortality rates have ranged $23-68 \%$ (15). Fortunately, our patient recovered after surgical and medical treatment and discharged with no important complication.

\section{CONCLUSION}

To our best knowledge, it is the first case of Acinetobacter endocarditis has ever been reported in Iran. Although MDR or XDR Acinetobacter baumannii is a life-threatening pathogen, especially in nosocomial setting and is a great concern, proper and timely treatment can be life-saving. In addition, more effective strategies and surveillance must be used to prevent the dissemination of Acinetobacter baumannii in the hospital.

\section{ACKNOWLEDGEMENTS}

All authors met four criteria for authorship contribution based on recommendations of the International Committee of Medical Journal Editors. No financial support.

\section{REFERENCES}

1. Fournier PE, Richet $\mathrm{H}$. The epidemiology and control of Acinetobacter baumannii in health care facilities. Clin Infect Dis 2006; 42:692-699.

2. Pires S, Parker D. Innate immune responses to Acinetobacter baumannii in the airway. J Interferon Cytokine Res 2019; 39:441-449.

3. Sturiale M, Corpina C, Sturiale L. Endocarditis duo to 
Acinetobacter baumannii. Int J Cardiol 2016; 209:161163.

4. Lahmidi I, Charmake D 3rd, Elouafi N, Bazid Z. Acinetobacter baumannii native valve infective endocarditis: a case report. Cureus 2020; 12(11): e11527.

5. Howard A, O`Donghue M, Feeney A, Sleator RD. Acinetobacter baumannii: an emerging opportunistic pathogen. Virulence 2012; 3:243-250.

6. Morris FC, Dexter C, Kostoulias X, Uddin MI, Peleg AY. The mechanisms of disease caused by Acinetobacter baumannii. Front Microbiol 2019; 10:1601.

7. Wong D, Nielsen TB, Bonomo RA, Pantapalangkoor P, Luna B, Spellberg B. Clinical and pathophysiological overview of Acinetobacter infections: a century of challenges. Clin Microbiol Rev 2017; 30:409-447.

8. Houghton T, Kaye GC, Meigh RE. An unusual case of infective endocarditis. Postgrad Med J 2002; 78:290291.

9. Chen Q, Cao H, Lu H, Qiu ZH, He JJ. Bioprosthetic tricuspid valve endocarditis caused by Acinetobacter baumannii complex, a case report and brief review of the literature. J Cardiothorac Surg 2015; 10:149.

10. Sturiale M, Corpina C, Sturiale L. Endocarditis due to Acinetobacter baumannii. Int J Cardiol 2016; 209:161-
163.

11. Kumar SS, Vengadassalapathy L, menon T. Prosthetic valve endocarditis caused by Acinetobacter baumannii complex. Indian J Pathol Microbiol 2008; 51:573.

12. Hoen B. Duval X. Clinical practice. Infective endocarditis. N Engl J Med 2013; 368:1425-1433.

13. Khoshnood S, Svari M, Abbasi Montazeri E, Frajzadeh Sheikh A. Survey on genetic diversity, biofilm formation, and detection of colistin resistance genes in clinical isolates of Acinetobacter baumannii. Infect Drug Resist 2020; 13:1547-1558.

14. Ezadi F, Jamali A, Heidari A, Javid N, Ardebili A. Heteroresistance to colistin in oxacillinases-producing carbapenem-resistant Acinetobacter baumannii clinical isolates from Gorgan, northern Iran. J Glob Antimicrob Resist 2020; 21:380-385.

15. Freire MP, de Oliveira Garcia D, Garcia CP, Campagnari Bueno MF, Camargo CH, Kono Magri ASG, et al. Bloodstreaminfection caused by extensively drug-resistant Acinetobacter baumannii in cancer patients: high mortality associated with delayed treatment rather than with the degree of neutropenia. Clin Microbiol Infect 2016; 22:352-358 